\title{
Moyamoya Syndrome in Post-Transplant Children with Type 1 Neurofibromatosis and Congenital Nephrotic Syndrome
}

\author{
Reyner Loza ${ }^{1,2}{ }^{*}$, Nathalie Rodriguez ${ }^{1}$, Angelica Ynguil ${ }^{1}$, Silvia Sotelo ${ }^{3}$ \\ ${ }^{1}$ Department of Pediatrics, Pediatric Kidney Transplant Unit, Cayetano Heredia National Hospital, Lima, Peru \\ ${ }^{2}$ Pediatrics Department, Alberto Hurtado School of Medicine, Cayetano Heredia Peruvian University, Lima, Peru \\ ${ }^{3}$ Department of Radiology, Cayetano Heredia Hospital, Lima, Peru \\ Email: ^reyner.loza@upch.pe
}

How to cite this paper: Loza, R., Rodriguez, N., Ynguil, A. and Sotelo, S. (2021) Moyamoya Syndrome in Post-Transplant Children with Type 1 Neurofibromatosis and Congenital Nephrotic Syndrome. Open Journal of Nephrology, 11, 459-466.

https://doi.org/10.4236/ojneph.2021.114038

Received: August 26, 2021

Accepted: October 26, 2021

Published: October 29, 2021

Copyright $\odot 2021$ by author(s) and Scientific Research Publishing Inc. This work is licensed under the Creative Commons Attribution International License (CC BY 4.0).

http://creativecommons.org/licenses/by/4.0/

\begin{abstract}
Background: Neurofibromatosis (NF) is a genetic disorder of the nervous system that affects the development and growth of neuronal tissues. It is characterized by the development of malignant and benign tumors of the peripheral nerves and meningiomas, and the clinical manifestations can vary, including cerebral vascular compromise that precipitates symptoms characteristic of Moyamoya syndrome. The behavior of a posttransplant child with type $1 \mathrm{NF}$ who presents with characteristics of Moyamoya syndrome and is a carrier of a WT1 genetic mutation is unknown, which is why the report was made. Case Report: A child with type I NF with a history of cryptorchidism, hypospadias, bilateral sensorineural hearing loss, and chronic renal failure was arising from a congenital nephrotic syndrome due to a WT1 gene mutation at 3 years of age began renal replacement therapy initially with chronic ambulatory peritoneal dialysis and hemodialysis for refractory peritonitis. $\mathrm{He}$ underwent two episodes of transient ischemic attack (TIA) characterized by right hemiparesis during hemodialysis therapy and during six months postkidney transplantation present a similar episode. Studies for other causes were negative, and imaging studies show characteristics of NF-related vasculitis, cortical lesions, and focal lesions in the left frontal subcortical white matter with annular enhancement and subcortical edema in the parasagittal region of the left parietal lobe, as well as a decrease in flow in the cortical branches of the middle cerebral artery were described. After four weeks, the clinical manifestations of hemiparesis completely disappeared without leaving any sequels continuing with the regular immunosuppression of the everolimus, mycophenolate and low dose steroids. Conclusion: These findings are suggestive of small and medium-sized vessel vasculitis compatible with Moya-
\end{abstract}


moya syndrome in child post-transplantation associated with arteriopathy by NF.

\section{Keywords}

Kidney Transplantation, Moyamoya Syndrome, Neurofibromatosis, Congenital Nephrotic Syndrome

\section{Introduction}

Neurofibromatosis (NF) is a genetic disorder of the nervous system that affects the development and growth of neuronal tissues.

This disorder is characterized by the development of various tumors, including neurofibromas, neurinomas, malignant and benign tumors of the peripheral nerves, and meningiomas. Accompanying skin changes and bone deformities are also common in NF [1].

However, the clinical manifestations can be variants that include cerebral vascular compromise that precipitates symptoms of Moyamoya syndrome.

Patients with Moyamoya vasculopathy, who also have well recognized associated conditions, are categorized as having the Moyamoya Syndrome, whereas patients with no known associated risk factors are said to have moyamoya disease.

The pathognomonic arteriographic findings are bilateral in moyamoya disease. The severity can differ between patients with unilateral findings who have the moyamoya syndrome, even if they have no other associated risk factors [2].

The evolution of a post-transplant child with type $1 \mathrm{NF}$ who presents with characteristics of Moyamoya syndrome and is a carrier of a WT1 genetic mutation is not very described, unknown, which is why the report was made.

\section{Case Presentation/Report}

In this article we will discuss the case of an eight-year-old male child carrier of multiple "café au lait" spots in addition to masses of $0.5 \mathrm{~cm}$ palpable in the paravertebral region.

This child diagnosed with NF type I and with a history of cryptorchidism, hypospadias, sensorineural hearing loss, bilateral and chronic renal failure derived from nephrotic syndrome birth. At 3 years old, he began renal replacement therapy, first with ambulatory peritoneal dialysis chronic and then he continued with hemodialysis due to refractory peritonitis.

During hemodialysis therapy at 6 years old he presented an episode of transient left occipital ischemia, the serologies for cytomegalovirus (CMV), Epstein-Barr virus, anti-DNA, complement $\mathrm{C} 3$, Anca $\mathrm{C}$ and $\mathrm{P}$ were negative.

Carotid Doppler ultrasound did not show any signs of stenosis. Protein C hypercoagulability studies showed a value of $82 \%(70 \%$ - 140\%). Protein S: $97 \%$ 
(60\% - 140\%). Antithrombin III level was normal. The study of the cause of kidney disease was a mutation of the WT1 Ex9 gene: 1390G > A (hetero); p. Asp443Asn (Courtesy of Research Laboratory Specialist Lead Sampson Laboratory University of Michigan).

At 8 years of age, he underwent a transplant from a living donor related to his father with a favorable outcome. He was initially prescribed tacrolimus, mycophenolate mofetil, and steroids. Eventually, tacrolimus was changed to Everolimus due to NF.

Six months after transplantation, he presented with signs of a Transient ischemic attack (TIA) cerebrovascular characterized by abrupt and right paresis and transient left central facial paralysis. In the clinical evaluation, Lisch nodules were found, and imaging studies using computerized axial tomography showed ischemia in the left cerebral hemisphere. Magnetic resonance imaging with cerebral angiography showed the axial section of the fluid attenuated inversion recovery (FLAIR) sequence: cortical signal hyperintensity in the left frontal lobe and an area of subcortical edema in the parasagittal region of the left parietal lobe (see Figure 1). Axial section in T1 sequence with gadolinium showed left frontal cortical enhancement and small focal lesions in the left frontal subcortical white matter with annular enhancement (see Figure 2). In the coronal section of the T2 sequence, hyperintensity of the left frontal cortical signal was observed (see Figure 3). Angioresonance showed a decrease in flow in the cortical branches of the middle cerebral artery on the left side associated with irregularity

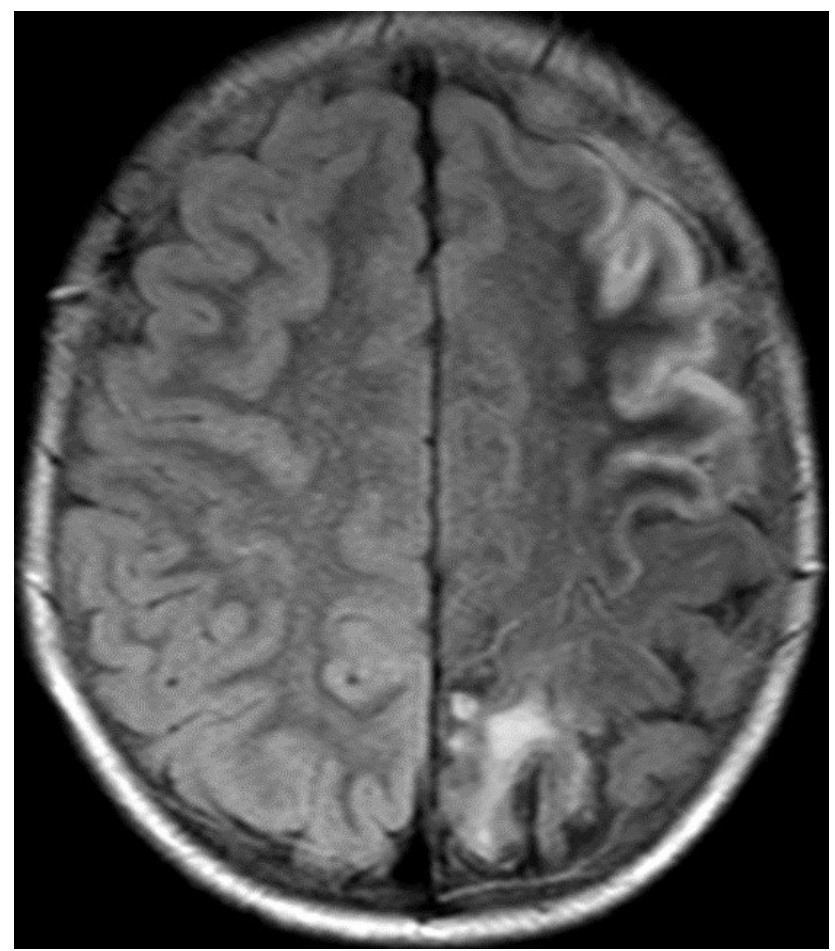

Figure 1. Axial section of the FLAIR sequence: cortical signal hyperintensity in the left frontal lobe and area of subcortical edema in the parasagittal region of the left parietal lobe. 


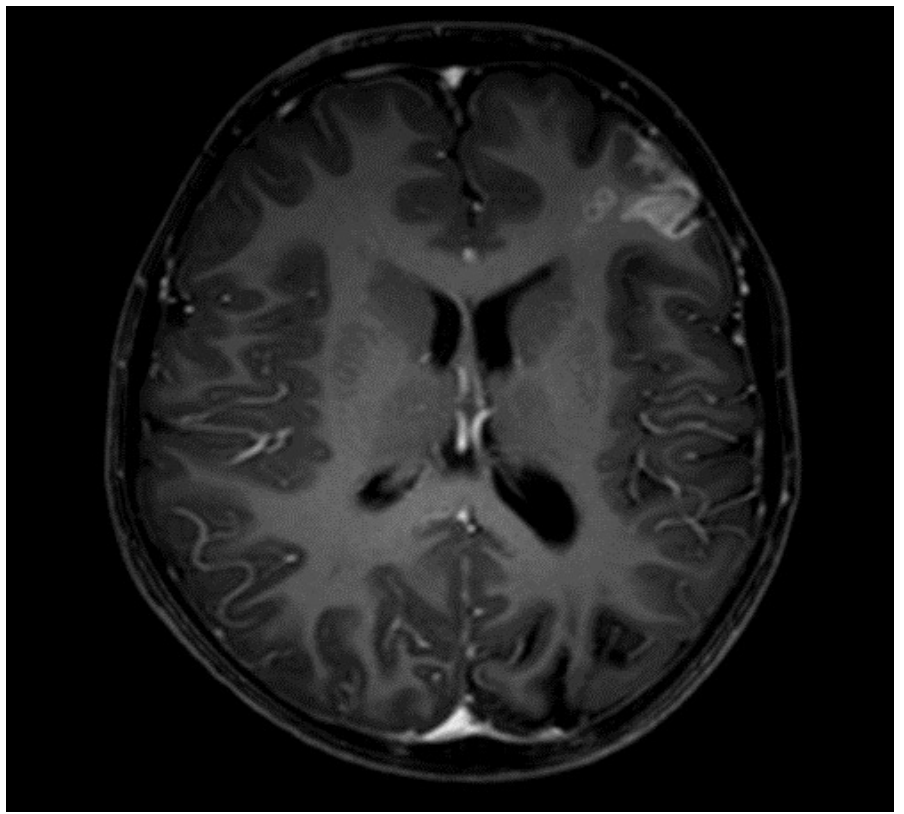

Figure 2. Axial section in T1 sequence with gadolinium: left frontal cortical enhancement and small focal lesions in the left frontal subcortical white matter with annular enhancement.

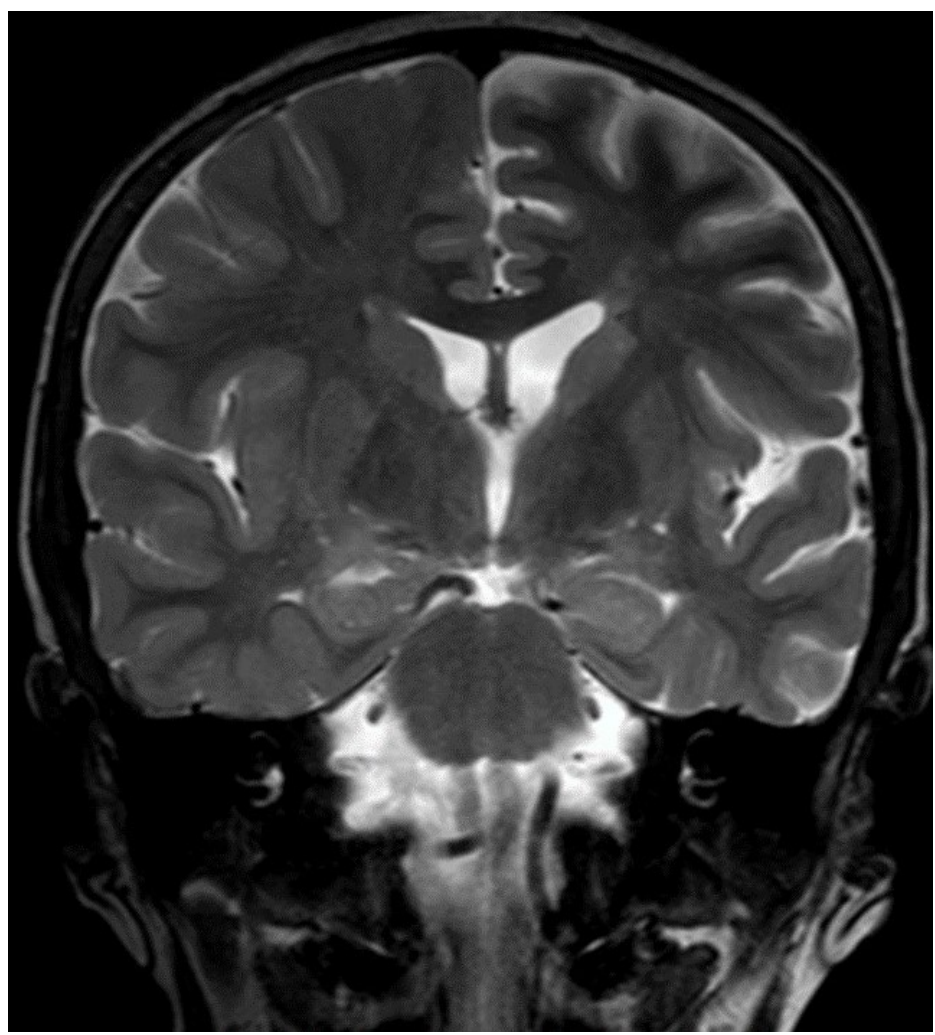

Figure 3. Coronal section in the T2 sequence: Hyperintensity of cortical signal in the left frontal.

and reduction of caliber in supraclinoid segments of the carotid arteries and of the left posterior cerebral artery irregularity of the cortical arteries in the left cerebral hemisphere (see Figure 4). Angiographic findings of moyamoya with 


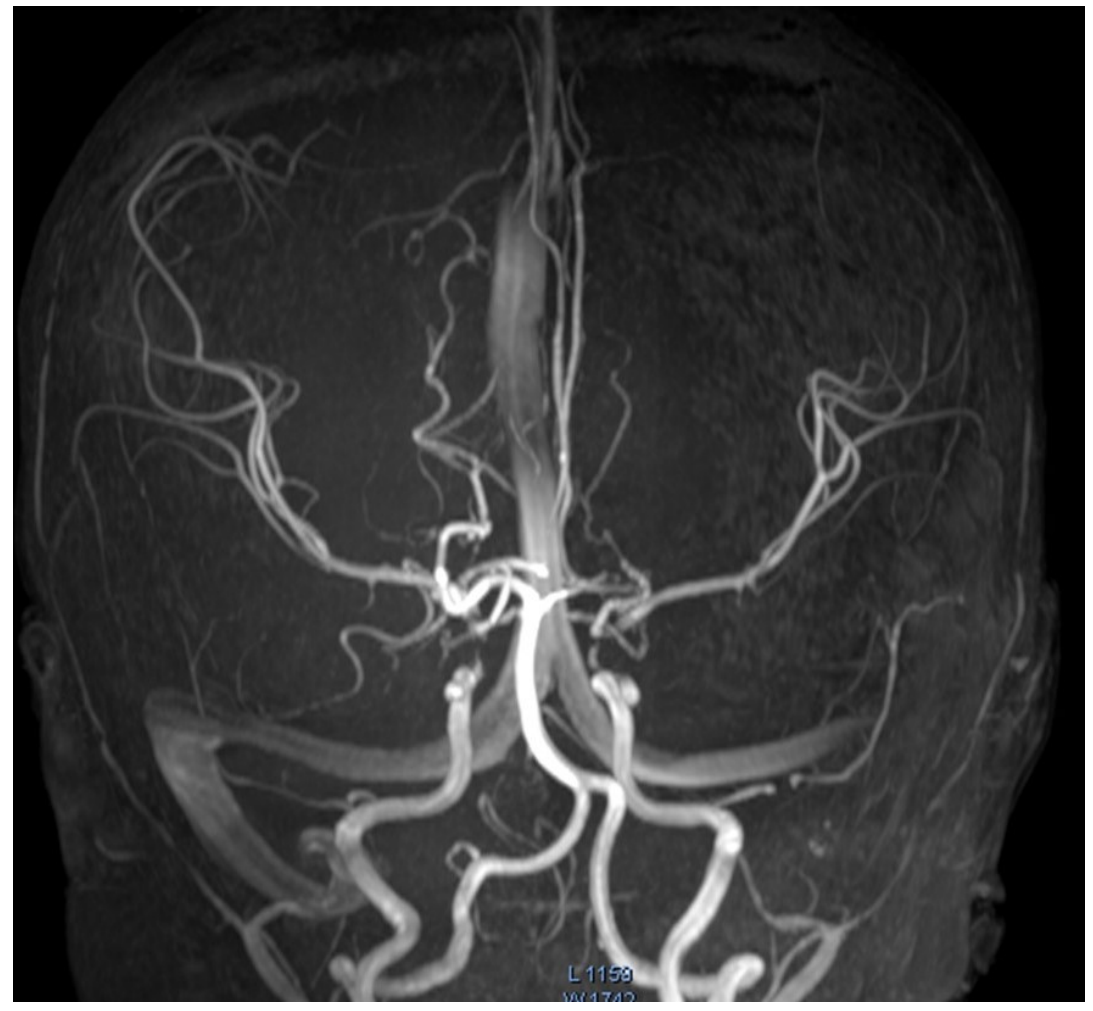

Figure 4. Decreased flow in the cortical branches of the left-sided middle cerebral artery. Poor flow in the left sigmoid sinus on the left side irregularity and decrease in caliber in supraclinoid segments of the carotid arteries and the left posterior cerebral irregularity of cortical arteries in the left cerebral hemisphere.

other disease compatible with the previously described on vascular alterations in Moyamoya syndrome associated with neurofibromatosis that are unilateral in nature. After four weeks, the clinical manifestations de hemiparesis completely disappeared without leaving any sequelae continuing with the usual immunosuppression of everolimus, mycophenolate, low-dose steroids, and acetylsalicylic acid as an antiplatelet agent.

\section{Discussion}

NF is a neurocutaneous syndrome that has the most distinctive clinical features of brown macules on the skin and neurofibromas. It is inherited in an autosomal dominant manner. It affects 1 in 3000 newborns, and more than $50 \%$ of cases correspond to novo mutations.

The responsible gene, NF1, located at 17q11.2, encodes a protein called neurofibromin, which is a tumor suppressor [1].

Cerebrovascular abnormalities in NF1 patients are seen in $4 \%-6 \%$ of children, but this usually does not cause ischemia since there are collaterals that supplement the blood supply [3]. The incidence is $0.086 / 100,000$ in USA, $0.54 / 100,000$ in Japan, and 0.03/100,000 in Europe.

Symptoms appear in the first-second decade of life; however, children with NF1 who develop Moyamoya syndrome are frequently asymptomatic. Children 
have a higher rate of completed strokes because of their immature verbal and reporting skills.

Symptoms of cerebral ischemia in moyamoya are typically associated with the regions of the brain supplied by the internal carotid arteries and middle cerebral arteries; these regions include the frontal, parietal, and temporal lobes. Hemiparesis, dysarthria, aphasia, and cognitive impairment are common.

Moyamoya syndrome is increasingly diagnosed worldwide and represents a major cause of childhood stroke in Western countries. It has been described in children with other medical conditions, such as sickle cell disease and Down syndrome [4] [5].

Moyamoya syndrome is a condition described in patients with NF and is much rarer in children after kidney transplant. The etiology is vasculopathy caused by a deficit in the production of neurofibrin, which would cause the proliferation of smooth muscle cells in the vessels in response to injury. This can result in the progressive obstruction of the arteries of the polygon of Willis in the region and the supraclinoid segment of the internal carotid arteries accompanied by collateral vascular circulation dependent on the external carotid and basilar arteries, as in the case reported here. There are few reports on the behavior of post-transplant children with neurofibromatosis [6] [7].

Angiographic changes associated with moyamoya are shared by a diverse collection of genetic and acquired conditions. There is a heterogeneity of the pathophysiological processes underlying these radiographic findings that reflect distinct clinical presentations.

The finding most suggestive of moyamoya on MRI is reduced flow voids in the internal, middle, and anterior cerebral arteries coupled with prominent flow voids through the basal ganglia and thalamus from moyamoya-associated collateral vessels. These findings are a diagnostic of moyamoya. In the case of our child, there was a decrease in flow in the cortical branches of the middle cerebral artery on the left side.

Regarding the mutation in our patient, the WT1 gene provides instructions to produce a protein necessary for the development of the kidneys and gonads (ovaries in women and testes in men) before birth.

The WT1 protein regulates the activity of other genes by binding to specific DNA regions. Based on this action, the WT1 protein is known as a transcription factor.

WT1 mutations can lead to three distinct clinical entities that are associated with steroid-resistant nephrotic syndrome (SNRE), glomerular disease: 1) Denys-Drash syndrome (DDS), 2) Frasier syndrome (SF), and 3) isolated nephrotic syndrome (ANS) [7] [8].

In more than $95 \%$ of cases with NS and WT1 mutations, the mutations are found in exon 8 or 9 of WT1. In the case of our patient, an isolated ANS is the etiology because there were no other associated clinical manifestations of kidney disease. However, its association with NF remains unknown [9]. 
Multiple genetic alterations are associated with chronic kidney disease. The vascular compromise characteristic of Moyamoya syndrome in child post-transplantation with NF and congenital nephropathy is one of the reasons we considered reporting this case.

\section{Conclusion}

These findings are suggestive of small and medium-sized vessel vasculitis compatible with Moyamoya syndrome in child post-transplantation associated with arteriopathy by NF.

\section{Consent}

The written consent of the patient was obtained for the publication of this clinical case and the use of the attached images.

\section{Author Contributions}

RL, NR, AY, wrote and edited the manuscript. SS collected all the images and their respective descriptions.

\section{Data Availability Statement}

Further information and requests for data should be directed to and will be fulfilled by the corresponding author Reyner Loza (reyner.loza@upch.pe).

\section{Conflicts of Interest}

The authors declare no conflicts of interest regarding the publication of this paper.

\section{References}

[1] Guillen Navarro, E., Ballesta Martínez, M.J. and Galan Gómez, E. (2010) Protocolo de seguimiento de la neurofibromatosis tipo 1. Protoc Diagn Pediatr, 1, 44-50.

[2] Scott, R.M. and Smith, E.R. (2009) Moyamoya Disease and Moyamoya Syndrome. The New England Journal of Medicine, 360, 1226-1237. https://doi.org/10.1056/NEJMra0804622

[3] Santoro, C. (2017) Moya Moya Syndrome in Children with Neurofibromatosis Type 1: Italian French Experience. American Journal of Medical Genetics, Part A, 173, 1521-1530.

[4] Parray, T., Martin, T.W. and Siddiqui, S. (2011) Moya Moya Disease: A Review of the Disease and Anesthetic Management. Journal of Neurosurgical Anesthesiology, 23, 100-109. https://doi.org/10.1097/ANA.0b013e3181f84fac

[5] Dlamini, N., Muthusami, P. and Amlie-Lefond, C. (2019) Childhood Moya Moya: Looking Back to the Future. Pediatric Neurology, 91, 11-19. https://doi.org/10.1016/j.pediatrneurol.2018.10.006

[6] Oates, A., Brennan, J., Slavotinek, A., Alsadah, A., Chow, D. and Lee, M.M. (2016) Challenges Managing End-Stage Renal Disease and Kidney Transplantation in a Child with MTFMT Mutation and Moyamoya Disease. Pediatric Transplantation, 
20, 1000-1003. https://doi.org/10.1111/petr.12758

[7] Merlo, C.A., Studer, S.M., Conte, J.V., Yang, S.C., Sonnett, J. and Orens, J.B. (2004) The Course of Neurofibromatosis Type 1 on Immunosuppression after Lung Transplantation: Report of Two Cases. Journal of Heart and Lung Transplantation, 23, 774-776. https://doi.org/10.1016/S1053-2498(03)00265-1

[8] Gersch, M.S. and Talor, Z. (2006) Focal Segmental Glomerular Sclerosis in a Patient with Neurofibromatosis Type I. American Journal of Kidney Diseases, 47, e17-e19. https://doi.org/10.1053/j.ajkd.2005.09.017

[9] Varyani, U.T., Shah, N.M., Shah, P.R., Kute, V.B., Balwani, M.R. and Trivedi, H.L. (2019) C1q Nephropathy in a Patient of Neurofibromatosis Type 1: A Rare Case Report. Indian Journal of Nephrology, 29, 125-127.

https://doi.org/10.4103/ijn.IJN 35317 